\title{
A Dirichlet Inhomogenous Boundary Value Problem for 1D Nonlinear Schrödinger Equation
}

\author{
Charles Bu \\ Department of Mathematics, Wellesley College, Wellesley, MA, USA \\ Email: cbu@wellesley.edu
}

How to cite this paper: $\mathrm{Bu}, \mathrm{C}$. (2022) A Dirichlet Inhomogenous Boundary Value Problem for 1D Nonlinear Schrödinger Equation. Journal of Applied Mathematics and Physics, 10, 656-660.

https://doi.org/10.4236/jamp.2022.103047

Received: February 9, 2022

Accepted: March 5, 2022

Published: March 8, 2022

Copyright $\odot 2022$ by author(s) and Scientific Research Publishing Inc. This work is licensed under the Creative Commons Attribution International License (CC BY 4.0).

http://creativecommons.org/licenses/by/4.0/

\begin{abstract}
Pure initial value problems for important nonlinear evolution equations such as nonlinear Schrödinger equation (NLS) and the Ginzburg-Landau equation (GL) have been extensively studied. However, many applications in physics lead to mathematical models where boundary data is inhomogeneous, e.g. in radio frequency wave experiments. In this paper, we investigate the mixed initial-boundary condition problem for the nonlinear Schrödinger equation $i u_{t}=u_{x x}-g|u|^{p-1} u, g \in R, p>3$ on a semi-infinite strip. The equation satisfies an initial condition and Dirichlet boundary conditions. We utilize semigroup theory to prove existence and uniqueness theorem of a strong local solution.
\end{abstract}

\section{Keywords}

Nonlinear Schrödinger Equation, Inhomogeneous Boundary Condition,

Local Existence and Uniqueness

\section{Introduction}

Inhomogenous boundary value problems often occur when an external force is applied to the time evolution of systems governed by nonlinear partial differential equations. Such forcing is often accomplished by imposing a boundary condition [1] [2]. In ionospheric modification experiments, one directs a radio frequency wave at the ionosphere. At the reflection point of the wave, a sufficient level of electron plasma waves is excited to make the nonlinear behavior important [3] [4]. This is described by the nonlinear Schrödinger equation with the cubic nonlinear term on a semi-infinite line. 


$$
\begin{gathered}
i u_{t}=u_{x x}+k|u|^{2} u \\
(x, t) \in R^{+} \times R^{+} \\
u(x, 0)=h(x), u(0, t)=g(t)
\end{gathered}
$$

where $k \in R^{+}$and $h(x), g(t)$ satisfy the necessary compatibility conditions $h(0)=g(0)$ with appropriate smoothness that $h \in H^{2}[0, \infty), g \in C^{1}[0, \infty)$. For more references about forced nonlinear systems such as NLS and KdV, see [5]-[11].

Although solutions to the free NLS satisfy various conservation laws, it is no longer the case when inhomogenous boundary condition is imposed. Consequently, Hamiltonian properties are lost and the nice Schrödinger group on $(-\infty, \infty)$ determined by $i q_{t}=q_{x x}$ is not available.

For (1.1) with either Dirichlet or Robin inhomogenous boundary condition, global existence and well-posedness for the forced NLS were proved in [12] [13].

For the following $1 \mathrm{D}$ nonlinear Schrödinger equation of a general nonlinearity $(g>0, p>3)$

$$
\begin{gathered}
i u_{t}=u_{x x}-g|u|^{p-1} u,(x, t) \in R^{+} \times R^{+} \\
u(x, 0)=h(x)
\end{gathered}
$$

with either Dirichlet or Robin Inhomogenous boundary conditions, global existence and well-posedness of a classical solution were proved in [14].

For the following NLS in $n$-dimensions with inhomogenous Dirichlet boundary data where the nonlinear term contributes to a positive term to the energy ( $\phi$ and $Q$ are given smooth functions):

$$
\begin{gathered}
i \partial_{t} u=\Delta u-f(u) \text { for } x \in \Omega \subset R^{n}, \\
u(x, 0)=\phi(x), \\
u(x, t)=Q(x, t) \text { for } x \in \partial \Omega,
\end{gathered}
$$

the existence of global solution of finite energy was proved in [15].

For a class of second-order nonlinear difference equations with Dirichlet boundary value problem, sufficient conditions on the existence of sign-changing solutions were obtained by using invariant sets of descending flow and variational methods [16].

In this paper, we will investigate a more general version of nonlinear Schrödinger equation $i u_{t}=u_{x x}-g|u|^{p} u$ when $x \in \Omega=[0, L], 0 \leq t<\infty$. The domain is a semi-infinite strip instead of a quarter plane. Dirichlet type inhomogeneous boundary conditions are imposed on the boundary. In next section, we use semigroup technique to prove the existence and uniqueness theorem for a local classical solution when initial and boundary data are smooth.

\section{Existence and Uniqueness Theorem}

We consider the following Dirichlet type inhomogenous boundary value problem for the 1D NLS: 


$$
\begin{gathered}
i u_{t}=u_{x x}-g|u|^{p-1} u, \\
x \in \Omega=[0, L], 0 \leq t<\infty, g \in R, p>3 \\
u(x, 0)=h(x), u(0, t)=Q_{1}(t), u(L, t)=Q_{2}(t) .
\end{gathered}
$$

We assume that $h(x) \in H^{2}(\Omega), Q_{1}(t) \in C^{1}(\Omega), Q_{2}(t) \in C^{1}(\Omega)$. In addition, the initial and boundary data satisfy the necessary compatibility conditions to ensure the existence of solution at $\partial \Omega$ and $t=0$.

We use the following substitution (note that $g>0$ is no longer required):

$$
u(x, t)=v(x, t)+\frac{x}{L} Q_{2}(t)+\frac{L-x}{L} Q_{1}(t)=v(x, t)+F(x, t)
$$

and convert (2.1) to

$$
i v_{t}+i F_{t}=v_{x x}-g|v+F|^{p-1}(v+F)
$$

This can be expressed as the following system (for some $a>0$ ):

$$
\begin{gathered}
v_{t}=-i v_{x x}-a v+K(t, v) \\
v(x, 0)=h(x)-F(x, 0), \\
v(0, t)=u(0, t)-F(0, t)=0, \\
v(L, t)=u(L, t)-F(L, t)=0 .
\end{gathered}
$$

where $K$ consists of all nonlinear terms of $v$ up to power of $p$ and $Q_{1}, Q_{1}^{\prime}, Q_{2}, Q_{2}^{\prime}$. We have the following existence of a unique classical solution.

Theorem 2.1. Let $A=-i D_{x}^{2}-a v$ with $D(A)=\left\{f \in H^{2}(\Omega) ; f(0)=0, f(L)=0\right\}$. Given $h \in H^{2}(\Omega)$, $v=u-\frac{x}{L} Q_{2}(t)-\frac{L-x}{L} Q_{1}(t)$ and $h(0)=Q_{1}(0), h(L)=Q_{2}(0)$, the equation (2.4) for $v$ has a unique classical solution $v \in C^{1}\left(\left[0, T_{M}\right), L^{2}\right) \cap C^{0}\left(\left[0, T_{M}\right), D(A)\right)$ with either $T_{M}=\infty$ or $\lim \|v(t)\|_{D(A)}=\infty$ as $t \rightarrow T_{M}$. Therefore, the equation (2.1) with Dirichlet boundray data has a unique classical solution $u \in C^{1}\left(\left[0, T_{M}\right), L^{2}\right) \cap C^{0}\left(\left[0, T_{M}\right), H^{2}\right)$ with either $T_{M}=\infty$ or $\lim \|u(t)\|_{H^{2}}=\infty$ as $t \rightarrow T_{M}$.

Proof. Define $W=W(\Omega)=\left\{u \in L^{2}(\Omega), u_{x x} \in L^{2}(\Omega)\right\}$ with norm $\|u\|_{W}^{2}=\|u\|_{2}^{2}+\left\|u_{x x}\right\|_{2}^{2}$. Then $W$ is complete and the mapping $u \mapsto u_{x}: W \rightarrow L^{2}$ is continuous. To check $A$ os a semigroup generator, we look at the resolvant $R_{\lambda}=(\lambda-A)^{-1}$ so consider $(\lambda-A) v=f$ with $v(0)=v(L)=0$ and $f \in L^{2}$. This means $\lambda v+i v^{\prime \prime}+a v=f$ and $v(0)=v(L)=0$. Since

$$
((\lambda-A) v, v)=(\lambda+a)\|v\|^{2}+i \int_{0}^{L} v^{\prime \prime} \bar{v} \mathrm{~d} x=(\lambda+a)\|v\|_{2}^{2}-i\left\|v^{\prime}\right\|_{2}^{2}
$$

we take the real parts and use Cauchy-Schwarz inequality to see that $(\lambda+a)\|v\|_{2} \leq\|(\lambda-A) v\|$. As we know, Cauchy-Schwarz inequality is considered one of the most important and widely used inequalities in mathematics (see [8] [17] for details).

By Hille-Yosida Theorem in functional analysis which characterizes the generators of strongly continuous one-parameter semigroups of linear operators on 
Banach spaces [17], $A$ generates a strongly continuous contraction semigroup $N(t)=\exp (A t)$ for any $a \geq 0$. Therefore (2.4) is converted into an integral equation:

$$
v(t)=N(t) v_{0}+\int_{0}^{t} N(t-s) K(s, v) \mathrm{d} s .
$$

Evidently $D(A)$ with the graph norm is a Hilbert space with norm equivalent to the $H^{2}$ norm. From Gagliardo-Nirenberg estimates, we see that $K$ is continuous in $t$ and locally Lipschitz in $D(A)$ uniformly on $[0, T]$. Applying Theorem 6.1.7 of [17], the local existence theorem is therefore proved.

\section{Conclusion}

Important evolution equations like the nonlinear Schrödinger equation and the Ginzburg-Landau equation have many physical applications when the boundary data is not zero. Studies on these problems contribute towards a better understanding of nature of the partial differential equations (PDEs). This paper is focused on a nonlinear Schrödinger equation posed in a semi-infinite strip. We apply semigroup theory used to prove that there exists a unique strong local solution. The PDE method employed here is a different approach to studying the NLS via inverse scattering transformation. There is some further research that could be done in this area, such as well-posedness of the system, existence of a global solution, and decay estimates and numerical simulation. We will continue our research and report any progress.

\section{Acknowledgements}

This research was supported by the William R. Kenan Jr. Professorship, the Brachman Hoffman Small Grant and a Wellesley College Faculty Award.

\section{Conflicts of Interest}

The author declares no conflicts of interest regarding the publication of this paper.

\section{References}

[1] Chu, C., Xiang, L. and Baransky, Y. (1983) Solitary Waves Induced by Boundary Motion. Communications on Pure and Applied Mathematics, 36, 495-504. https://doi.org/10.1002/cpa.3160360407

[2] Kaup, D. (1984) Forced Integrable Systems. In: Rogers, C. and Moodie, T., Eds., Wave Phenomena: Modern Theory and Applications, Elsevier, Amsterdam, 163-174. https://doi.org/10.1016/S0304-0208(08)71262-3

[3] Sheerin, J., et al. (1982) Solitons and Ionospheric Modification. Journal of Atmospheric and Terrestrial Physics, 44, 1043-1048. https://doi.org/10.1016/0021-9169(82)90016-2

[4] Zakharov, V. and Shabat, A. (1972) Exact Theory of Two-Dimensional Self-Focusing and One-Dimensional Self-Modulation of Waves in Nonlinear Media. Soviet Physics-JETP, 34, 62-69. 
[5] Craig, W. and Wayne, C. (1993) Newton's Method and Periodic Solutions of Nonlinear Wave Equations. Communications on Pure and Applied Mathematics, 46, 14091498. https://doi.org/10.1002/cpa.3160461102

[6] Craig, W., Kappeler, T. and Strauss, W. (1992) Grain of Regularity for Equations of KdV Type. Annales de P Institut Henri Poincaré C, Analyse non linéaire, 9, 147-186. https://doi.org/10.1016/s0294-1449(16)30243-8

[7] Dafermos, C. (1970) Wave Equations with Weak Damping. SIAM Journal on Applied Mathematics, 18, 759-767. https://doi.org/10.1137/0118068

[8] Strauss, W. (1989) Nonlinear Wave Equations. CBMS (AMS), Providence. https://doi.org/10.1090/cbms/073

[9] Jones, C., Gardner, R. and Kapitula, T. (1980) Stability of Travelling Waves for Nonconvex Scalar Viscous Conservation Laws. Communications on Pure and Applied Mathematics, 46, 505-526. https://doi.org/10.1002/cpa.3160460404

[10] Jones, C. (1988) Standing Waves of Nonlinear Schrödinger Equations: Existence and Stability, Material Instabilities in Continuum Mechanics (Edinburgh, 1985-86). Oxford University Press, Oxford, 197-216.

[11] Yan, G. (1995) Singular Solutions of the Vlasov-Maxwell System on a Half Line. Archive for Rational Mechanics and Analysis, 43, 241-304. https://doi.org/10.1007/BF00382888

[12] Carroll, R. and Bu, C. (1991) Solutions of the Forced Nonlinear Schrödinger Equation (NLS) Using PDE Techniques. Applicable Analysis, 41, 33-51. https://doi.org/10.1080/00036819108840015

[13] Bu, C. (1992) On Well-Posedness of the Forced Nonlinear Schrödinger Equation. Applicable Analysis, 46, 219-239. https://doi.org/10.1080/00036819208840122

[14] Bu, C. (2000) Nonlinear Schrödinger Equation on the Semi-Infinite Line. Chinese Annals of Mathematics, Series A, 21, 437-448.

[15] Strauss, W. and Bu, C. (2001) An Inhomogeneous Boundary Value Problem for Nonlinear Schrödinger Equations. Journal of Differential Equations, 173, 79-91. https://doi.org/10.1006/jdeq.2000.3871

[16] Long, Y. and Zeng, B. (2017) Sign-Changing Solutions for Discrete Dirichlet Boundary Value Problem. Journal of Applied Mathematics and Physics, 5, 2228-2243. https://doi.org/10.4236/jamp.2017.511182

[17] Pazy, A. (1983) Semigroups of Linear Operators and Applications to PDE. Springer, New York. https://doi.org/10.1007/978-1-4612-5561-1 